\title{
Bunching of fluxons by Cherenkov radiation in Josephson multilayers
}

\author{
E. Goldobin* \\ Institute of Thin Film and Ion Technology (ISI), Research Center Jülich GmbH (FZJ), D-52425 Jülich, Germany \\ B. A. Malomed \\ Department of Interdisciplinary Studies, Faculty of Engineering, Tel Aviv University, 69978 Tel Aviv, Israel \\ A. V. Ustinov \\ Physikalisches Institut III, Universität Erlangen Nürnberg, D-91054 Erlangen, Germany
}

(Received 3 February 2000)

\begin{abstract}
A single magnetic fluxon moving at a high velocity in a Josephson multilayer (e.g., high-temperature superconductor such as BSCCO) can emit electromagnetic waves (Cherenkov radiation), which leads to the formation of stable dynamic states consisting of several bunched fluxons. We find such bunched states in numerical simulations in the simplest cases of two and three coupled junctions. At a given driving current, several different bunched states are stable and move at velocities that are higher than the corresponding single-fluxon velocity. These and some of the more complex higher-order bunched states and transitions between them are investigated in detail.
\end{abstract}

\section{INTRODUCTION}

In recent years, a great deal of attention has been attracted to different kinds of solid-state multilayered systems, e.g., artificial Josephson and magnetic multilayers, hightemperature superconductors (HTSs) and perovskites, to name just a few. Multilayers are attractive because it is often possible to multiply a physical effect achieved in one layer by $N$ (and sometimes by $N^{2}$ ), where $N$ is the number of layers. This can be exploited for fabrication of solid-state devices. In addition, multilayered solid-state systems show a variety of physical phenomena which result from the interaction between individual layers.

In this article we focus on Josephson multilayers, the simplest example of which is a stack consisting of just two long Josephson junctions (LJJs). The results of our consideration can be applied to intrinsically layered HTS materials, ${ }^{1}$ since the Josephson-stack model has proved to be appropriate for these structures. $^{2-4}$

In earlier papers ${ }^{5-8}$ it was shown that, in some cases, a fluxon (Josephson vortex) moving in one of the layers of the stack may emit electromagnetic (plasma) waves by means of the Cherenkov mechanism. The fluxon together with its Cherenkov radiation has the profile of a traveling wave, $\phi(x-u t)$, having an oscillating gradually decaying tail. Such a wave profile generates an effective potential for another fluxon which can be added into the system. If the second fluxon is trapped in one of the minima of this traveling potential, we can get a bunched state of two fluxons. In such a state, two fluxons can stably move at a small constant distance from one another, which is not possible otherwise. Fluxons of the same polarity usually repel each other, even being located in different layers.

Similar bunched states were already found in discrete Josephson transmission lines, ${ }^{9}$ as well as in long Josephson junctions with the so-called $\beta$ term due to the surface impedance of the superconductor. ${ }^{10-12}$ The dynamics of conven- tional LJJs is described by the sine-Gordon equation which does not allow the fluxon to move faster than the Swihart velocity and, therefore, the Cherenkov radiation never appears. In both cases mentioned above (the discrete system or the system with the $\beta$ term), the perturbation of the sineGordon equation results in a modified dispersion relation for Josephson plasma waves and the appearance of an oscillating tail. This tail, in turn, results in an attractive interaction between fluxons, i.e., bunching. Nevertheless, the mere presence of an oscillating tail is not a sufficient condition for bunching.

In this paper, we investigate the problem of fluxon bunching in a system of two and three inductively coupled junctions with a primary state $[1 \mid 0]$ (one fluxon in the top junction and no fluxon in the bottom one) or $[0|1| 0]$ (a fluxon only in the middle junction of a three-fold stack). We show that bunching is possible for some fluxon configurations and specific range of parameters of the system. In addition, it is found that the bunched states radiate less than single-fluxon states, and therefore can move with a higher velocity. Section II presents the results of numerical simulations; in Sec. III we discuss the obtained results and the feasibility of the experimental observation of bunched states. We also derive a simple analytical expression which shows the possibility of the existence of bunched states. Section IV concludes the work.

\section{NUMERICAL SIMULATIONS}

The system of equations which describes the dynamics of Josephson phases $\phi^{A, B}$ in two coupled $\mathrm{LJJ}^{A}$ and $\mathrm{LJJ}^{B}$ is ${ }^{13,14}$

$$
\begin{aligned}
& \frac{\phi_{x x}^{A}}{1-S^{2}}-\phi_{t t}^{A}-\sin \phi^{A}-\frac{S}{1-S^{2}} \phi_{x x}^{B}=\alpha \phi_{t}^{A}-\gamma, \\
& \frac{\phi_{x x}^{B}}{1-S^{2}}-\phi_{t t}^{B}-\frac{\sin \phi^{B}}{J}-\frac{S}{1-S^{2}} \phi_{x x}^{A}=\alpha \phi_{t}^{B}-\gamma,
\end{aligned}
$$


where $S(-1<S<0)$ is a dimensionless coupling constant, and $J=j_{c}^{A} / j_{c}^{B}$ is the ratio of the critical currents, while $\alpha$ and $\gamma=j / j_{c}^{A}$ are the damping coefficient and normalized bias current, respectively, which are assumed to be the same in both LJJs. It is also assumed that other parameters of the junctions, such as the effective magnetic thicknesses and capacitances, are the same. As has been shown earlier, ${ }^{6,7}$ the Cherenkov radiation in a twofold stack may take place only if the fluxon is moving in the junction with smaller $j_{c}$. We suppose in the following that the fluxon moves in $\mathrm{LJJ}^{A}$, which implies $J<1$.

In the case $N=3$, we impose the symmetry condition $\phi^{A} \equiv \phi^{C}$, which is natural when the fluxon moves in the middle layer, and, thus, we can rewrite equations from Ref. 13 in the form

$$
\begin{aligned}
& \frac{\phi_{x x}^{A}}{1-2 S^{2}}-\phi_{t t}^{A}-\sin \phi^{A}-\frac{S \phi_{x x}^{B}}{1-2 S^{2}}=\alpha \phi_{t}^{A}-\gamma, \\
& \frac{\phi_{x x}^{B}}{1-2 S^{2}}-\phi_{t t}^{B}-\sin \phi^{B}-\frac{2 S \phi_{x x}^{A}}{1-2 S^{2}}=\alpha \phi_{t}^{B}-\gamma .
\end{aligned}
$$

Note the factor 2 in the last term on the left-hand side (LHS) of Eq. (4). In the case of three coupled LJJs, we assume $J$ $=1$, since for more than two coupled junctions the Cherenkov radiation can be obtained for a uniform stack with equal critical currents. $^{7}$

\section{A. Numerical technique}

The numerical procedure works as follows. For a given set of LJJ parameters, we compute the current-voltage characteristic (IVC) of the system, i.e., $\bar{V}^{A, B}(\gamma)$. To calculate the voltages $\bar{V}^{A, B}$ for fixed values of $\gamma$, we simulate the dynamics of the phases $\phi^{A, B}(x, t)$ by solving Eqs. (1) and (2) for $N=2$ or Eqs. (3) and (4) for $N=3$, using the periodic boundary conditions

$$
\begin{gathered}
\phi^{A, B}(x=L)=\phi^{A, B}(x=0)+2 \pi N^{A, B}, \\
\phi_{x}^{A, B}(x=L)=\phi_{x}^{A, B}(x=0),
\end{gathered}
$$

where $N^{A, B}$ is the number of fluxons trapped in $\mathrm{LJJ}^{A, B}$. In order to simulate a quasi-infinite system, we have chosen annular geometry with the length (circumference) of the junction $L=100$.

To solve the differential equations, we use an explicit method [expressing $\phi^{A, B}(t+\Delta t)$ as a function of $\phi^{A, B}(t)$ and $\left.\phi^{A, B}(t-\Delta t)\right]$, treating $\phi_{x x}$ with a five-point, $\phi_{t t}$ with a three-point, and $\phi_{t}$ with a two-point symmetric finitedifference scheme. The spatial and time steps used for the simulations were $\delta x=0.025$ and $\delta t=0.00625$. After the simulation of the phase dynamics for $T=10$ time units, we calculate the average dc voltages $\bar{V}^{A, B}$ for this time interval as

$$
\bar{V}^{A, B}=\frac{1}{T} \int_{0}^{T} \phi_{t}^{A, B}(t) d t=\frac{\phi^{A, B}(T)-\phi^{A, B}(0)}{T} .
$$

The dc voltage at point $x$ can be defined as the average number of fluxons (the flux) passed through the junction at this point. Since the average fluxon density is not singular in any point of the junction (otherwise the energy will grow infinitely), we conclude that the average dc voltage is the same for any point $x$. Therefore, for faster convergence of our averaging procedure, we can additionally average the phases $\phi^{A, B}$ in Eq. (7) over the length of the stack.

After the values of $\bar{V}^{A, B}$ were found as per Eq. (7), the evolution of the phases $\phi^{A, B}(x, t)$ is simulated further during $1.1 T$ time units; the dc voltages $\bar{V}^{A, B}$ are calculated for this new time interval and compared with the previously calculated values. We repeat such iterations further, increasing the time interval by a factor of 1.1 until the difference in dc voltages $\left|\bar{V}\left(1.1^{n+1} T\right)-\bar{V}\left(1.1^{n} T\right)\right|$ obtained in two subsequent iterations becomes less than an accuracy $\delta V=10^{-4}$. The particular factor of 1.1 was found to be quite optimal and to provide for fast convergence, as well as a more efficient averaging of low harmonics on each subsequent step. A very small value of this factor, e.g., 1.01 (recall that only the values greater than 1 have meaning), may result in a very slow convergence in the case when $\phi(t)$ contains harmonics with period $\geqslant T$. Large values of the factor, e.g., $\geqslant 2$, would consume a lot of CPU time already during the second or third iteration and, hence, are not good for practical use.

Once the voltage averaging for current $\gamma$ is complete, the current $\gamma$ is increased by a small amount $\delta \gamma=0.005$ to calculate the voltages at the next point of the IVC. We use a distribution of the phases (and their derivatives) achieved in the previous point of the IVC as the initial distribution for the following point.

A further description of the software used for simulations can be found in Ref. 15.

\section{B. Two coupled junctions}

For simulations we chose the following parameters of the system: $S=-0.5$ to be close to the limit of intrinsically layered HTSs, $J=0.5$ to let the fluxon accelerate above the $\bar{c}_{-}$ and develop Cherenkov radiation tail. The velocity $\bar{c}_{-}$is the smallest of the Swihart velocities of the system. It characterizes the propagation of the out-of-phase mode of Josephson plasma waves. The value of $\alpha=0.04$ is chosen somewhat higher than, e.g., in $\left(\mathrm{Nb}-\mathrm{Al}-\mathrm{AlO}_{x}\right)_{N}-\mathrm{Nb}$ stacks. This choice is dictated by the need to keep the quasi-infinite approximation valid and satisfy the condition $\alpha L \gg 1$. A smaller $\alpha$ requires a very large $L$ and, therefore, unaffordably long simulation times. So we made a compromise and chose the above $\alpha$ value.

First, we simulated the IVC $u(\gamma)$ in the [1|0] state by sweeping $\gamma$ from 0 up to 1 and making snapshots of the phase gradients at every point of the IVC. This IVC is shown in Fig. 1(a), and the snapshot of the phase gradient at $\gamma$ $=0.3$ is presented in Fig. 1(b). As one can see, the Cherenkov radiation tail, which is present for $u>\bar{c}_{-}$, has a sequence of minima where the second fluxon may be trapped.

\section{1. $[1+1 \mid 0]$ state}

In order to create a two-fluxon bunched state and check its stability, we used the following "solution-engineering" procedure. By taking a snapshot of the phase profiles $\phi_{A, B}(x)$ at 

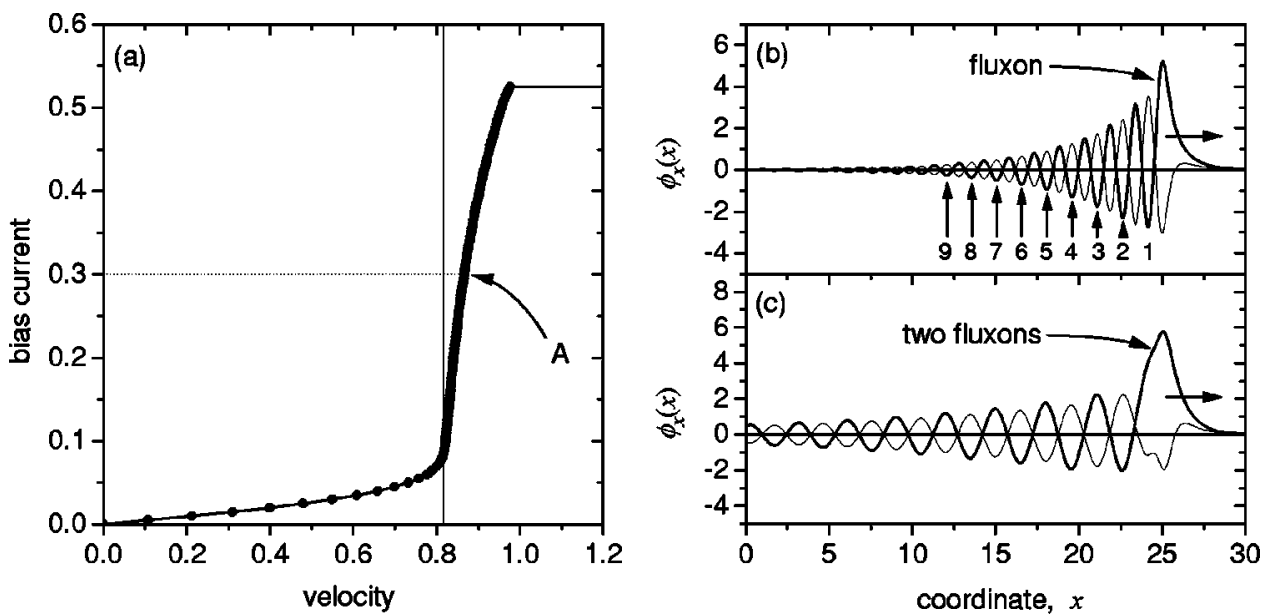

FIG. 1. (a) The current-velocity characteristic $u(\gamma)$ for the fluxon moving in the [1|0] state (from left to right). (b) The profiles of the phase gradients $\phi_{x}^{A, B}(x)$ in the state [1|0] at $\gamma=0.3$, corresponding to the bias point $A$ shown in fig. (a). The Cherenkov tail, present at $u>\bar{c}_{-} \approx 0.817$, has a set of minima where the second fluxon can be trapped. (c) The profiles of $\phi_{x}^{A, B}(x)$ in the state [2|0] at the same value $\gamma=0.3$ as (b). Two fluxons shown in Fig. (c) are almost undistinguishable.

the bias value $\gamma_{0}=0.3$, we constructed an ansatz for the bunched solution in the form

$$
\phi_{A, B}^{\text {new }}(x)=\phi_{A, B}(x)+\phi_{A, B}(x+\Delta x),
$$

where $\Delta x$ is chosen so that the center of the trailing fluxon is placed at one of the minima of the Cherenkov tail. For example, to trap the trailing fluxon in the first, second, and third wells, we used $\Delta x=0.9, \Delta x=2.4$, and $\Delta x=3.9$, respectively. The phase distribution (and derivatives), constructed in this way, were used as the initial condition for solving Eqs. (1) and (2) numerically. As the system relaxed to the desired state $[1+1 \mid 0]$, we further traced the $u(\gamma)$ curve, varying $\gamma$ down to 0 and up to 1 .

We accomplished this procedure for a set of $\Delta x$ values, trying to trap the second fluxon in every well. Figure 1(c) shows that a stable, tightly bunched state of two fluxons is indeed possible. Actually, all the $[1+1 \mid 0]$ states obtained this way have been found to be stable, and we were able to trace their IVCs up and down, starting from the initial value of the bias current $\gamma=0.3$. For the case when the trailing fluxon is trapped in the first, second, and third minima, such IVCs are shown in Fig. 2.

The most interesting feature of these curves is that they correspond to the velocity of the bunched state that is higher than that of the $[1 \mid 0]$ state, at the same value of the bias current. Comparing solutions shown in Figs. 1(b) and 1(c), we see that the amplitude of the trailing tail is smaller for the bunched state. This circumstance suggests the following explanation of the fact that the observed velocity is higher in the state $[1+1 \mid 0]$ than in the single-fluxon one. Because the driving forces acting on two fluxons in the bunched and unbunched states are the same, the difference in their velocities can be attributed only to the difference in the friction forces. The friction force acting on the fluxon in one junction is

$$
F_{\alpha}=\alpha \int_{-\infty}^{+\infty} \phi_{x} \phi_{t} d x
$$

and the same holds for the other junction. By just looking at Figs. 1(b) and 1(c) it is rather difficult to tell in which case the friction force is larger, but accurate calculations using Eq. (9) and profiles from Figs. 1(b) and 1(c) show that the friction force acting on two fluxons with the tails shown in Fig. 1(b) is somewhat higher than that for Fig. 1(c). This result is not surprising if one recalls that, to create the bunched state, we have shifted the $[1 \mid 0]$ state by about half of the tail oscillation period relative to the other singlefluxon state. Due to this, the tails of the two fluxons add up out of phase and partly cancel each other, making the tail's amplitude behind the fluxon in the bunched state lower than that in the $[1 \mid 0]$ state.

From Fig. 2 it is seen that every bunched state exists in a certain range of values of the bias current. If the current is decreased below some threshold value, fluxons dissociate and start moving apart, so that the interaction between them becomes exponentially small. When the trailing fluxon sits in a minimum of the Cherenkov tail sufficiently far from the leading fluxon, the IVC corresponding to this bunched state is almost undistinguishable from that of the $[1 \mid 0]$ state, as

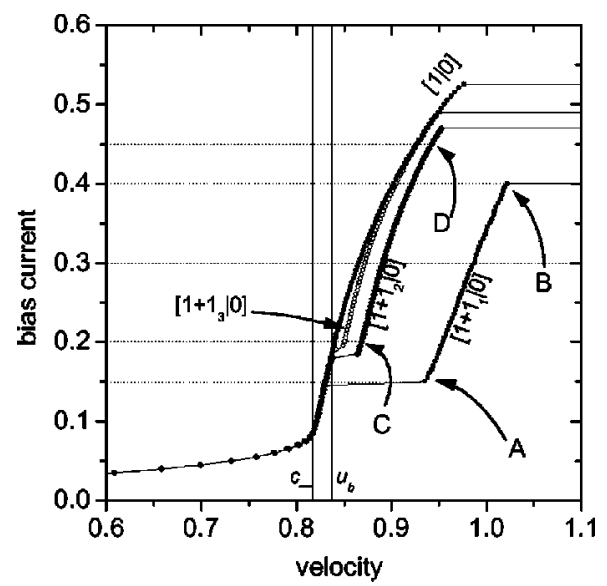

FIG. 2. Current-velocity characteristics of different bunched states [2|0]: the second fluxon is trapped in the first minimum of the tail (state $\left[1+1_{1} \mid 0\right]$ ), the second minimum (state $\left[1+1_{2} \mid 0\right]$ ), and the third minimum (state $\left[1+1_{3} \mid 0\right]$ ). The $\gamma(u)$ curve for the $[1 \mid 0]$ state is shown for comparison. The phase-gradient profiles corresponding to bias points $A-D$ are shown in Fig. 3 . 


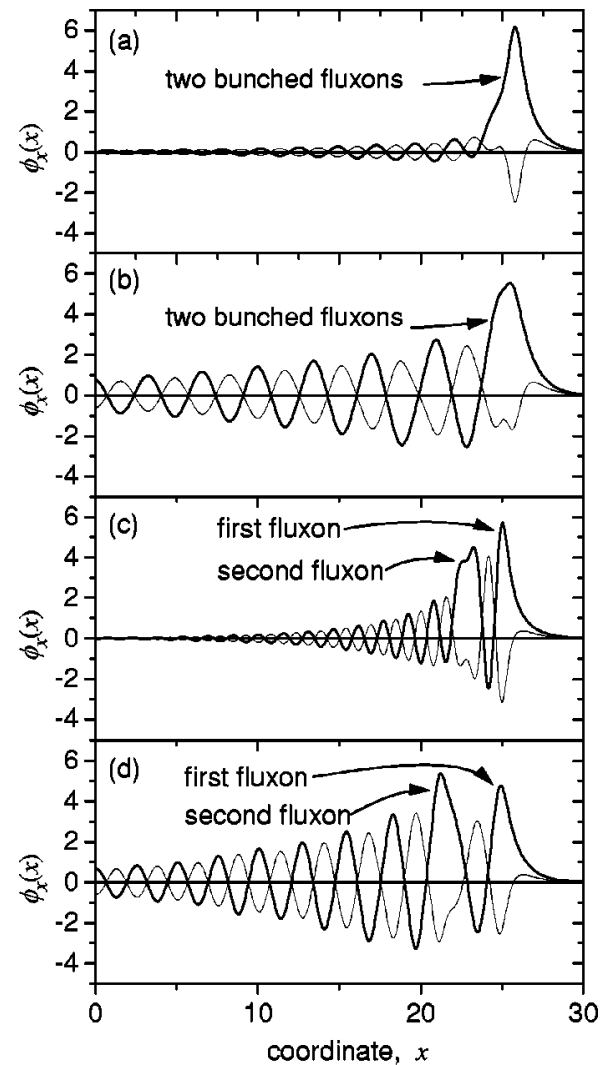

FIG. 3. The profiles of the phase gradients $\phi_{x}^{A, B}(x)$ in the [2|0] states at bias points $A-D$ marked in Fig. 2 .

the two fluxons approach the limit when they do not interact. We have found that IVCs for $M>3$, where $M$ is the potential well's number, are indeed almost identical to that of the $[1 \mid 0]$ state. In contrast to the bunching of fluxons in discrete $\mathrm{LJJs}_{\mathrm{s}}{ }^{9}$ the transitions from one bunched state to another with different $M$ do not take place in our system. Thus, we can say that the current range of a bunched state with smaller $M$ "eclipses" the bunched states with larger $M$.

The profiles of solutions found for various values of the bias current are shown in Fig. 3. We notice that at the bottom of the step corresponding to the bunched state the radiation tail is much weaker and fluxons are bunched tighter. This is a direct consequence of the fact that at lower velocities the radiation wavelength and the distance between minima become smaller, and so does the distance between the two fluxons. At a low bias current, the radiation wavelength and, hence, width of the potential wells become very small and incommensurable with the fluxon's width. Therefore, the fluxon does not fit into the well and the bunched states virtually disappear.

\section{2. $[1 \mid 1]$ state}

The initial condition for this state was constructed in a similar fashion to the $[1+1 \mid 0]$ one, but now using a crosssum of the shifted and unshifted solutions:

$$
\phi_{A, B}^{\text {new }}(x)=\phi_{A, B}(x)+\phi_{B, A}(x+\Delta x) .
$$

If for the $[1+1 \mid 0]$ state $\Delta x$ were $\approx\left(\lambda-\frac{1}{2}\right) M, M=1,2 \ldots$, then in the [1|1] state we have to take $\Delta x \approx \lambda M$. We can also take $M=0$, i.e., $\Delta x=0$, which corresponds to the de- generate case of the in-phase [1|1] state. The stability of this state was investigated in detail analytically by GrønbechJensen and co-workers, ${ }^{16}$, and is outside the scope of this paper.

Our efforts to create a bound state [1|1] using the phase in the form (10) with $M=1,2 \ldots$ have not led to any stable configuration of bunched fluxons with $\Delta x \neq 0$.

\section{Higher-order states}

Looking at the phase gradient profiles shown in Fig. 3, one notes that these profiles are qualitatively very similar to the original profile of the soliton with a radiation tail behind it [see Fig. 1(b)], with the only difference being that there are two bunched solitons with a tail. So we can try to construct two pairs of bunched fluxons moving together, i.e., get a $[2+2 \mid 0]$ bunched state. As before, the trapping of the trailing pair is possible in one of the minima of the tail generated by the leading pair. To construct such a double-bunched state we employ the initial conditions obtained using Eq. (8) at the bias point $\gamma_{0}=0.3$, using the steady phase distribution obtained for the $[2 \mid 0]$ state at $\gamma_{0}=0.3$. The shift $\Delta x$ was chosen in such a way that a pair of fluxons fits into one of the minima of the tail. We note that in this case we needed to vary $\Delta x$ a little bit before we achieved trapping of the trailing pair in a desired well.

Simulations show that the obtained $[2+2 \mid 0]$ states are stable and demonstrate an even higher velocity of the whole four-fluxon aggregate. The corresponding IVCs and profiles are shown in Figs. 4(a) and 4(b), respectively. Note that at $\gamma<0.22$ the bunched state $[2+2 \mid 0]$ splits first into a $[1$ $\left.+1_{2}+1_{3}+1_{3} \mid 0\right]$ state (the subscripts denote the well's number $M$, counting from the previous fluxon), and at still lower bias current $\gamma<0.2$, they split into two independent [1 $\left.+1_{2} \mid 0\right]$ and $\left[1+1_{5} \mid 0\right]$ states. This two states move with slightly different velocities and can collide with each other due to the periodic nature of the system. As a result of the collisions, these states ultimately undergo a transformation into two independent $\left[1+1_{5} \mid 0\right]$ states. As the bias decreases below $\approx 0.1$, the velocity $u$ becomes smaller than $\bar{c}_{-}$and the Cherenkov radiation tails disappear. At this point, each of the $\left[1+1_{5} \mid 0\right]$ states smoothly transforms into two independent $[1 \mid 0]$ states. The interaction between these states is exponentially small, with a characteristic length $\sim 1$ (or $\lambda_{J}$ in physical units). We note that the interaction between kinks in the region $u>\bar{c}_{-}$, where they have tails, also decreases exponentially, but with a larger characteristic length $\sim \alpha^{-1}$.

The procedure of constructing higher-order bunched states can be performed using different states as "building blocks.' In particular, we also tried to form the $[2+1 \mid 0]$ bunched state. Note that if two different states are taken as building blocks, we need to match their velocities and, hence, the wavelengths of the tail. Thus, we have to combine two states at the same velocity, rather than at the same bias current. Since different states have their own velocity ranges, it is not always possible. As an example, we have constructed a $[2+1 \mid 0]$ state out of a $[2 \mid 0]$ state at $\gamma=0.15$ and a $[1 \mid 0]$ state at $\gamma=0.45$ using an ansatz similar to Eq. (8). These states have approximately the same velocity $u \approx 0.95$ (see Fig. 2). The constructed state was simulated, starting from the points $\gamma=0.3$ and $\gamma=0.35$, tracing the IVC up and down as before. Depending on the bias current the system 

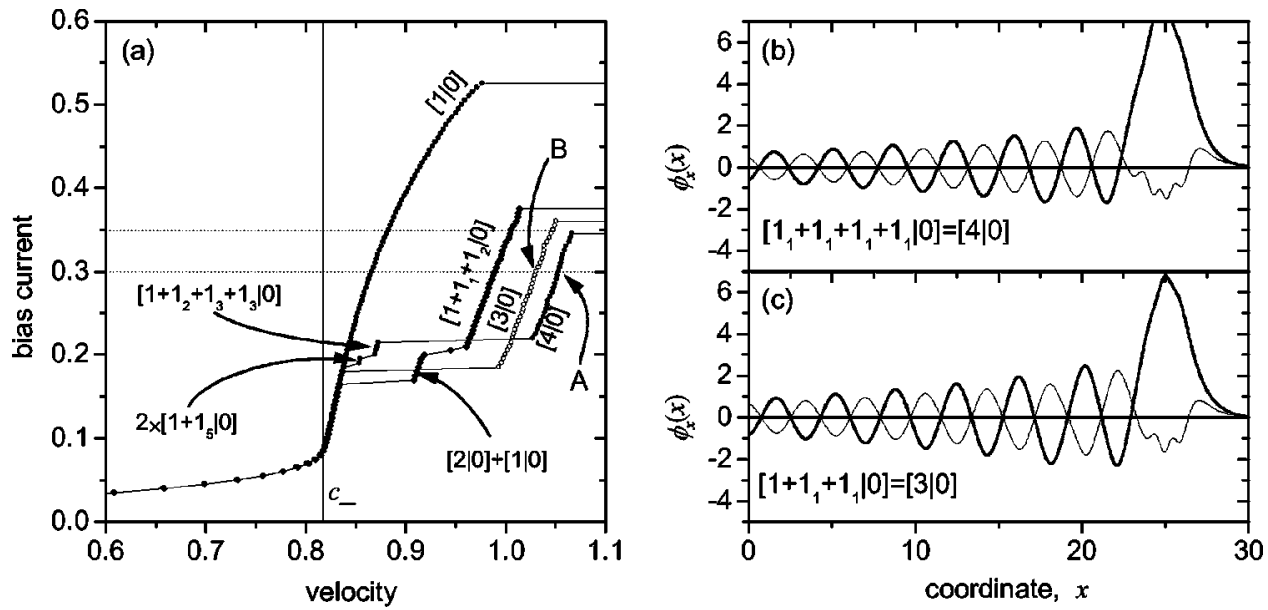

FIG. 4. (a) Current-velocity characteristics of the bunched states $[4 \mid 0],[3 \mid 0]$, and $[2+1 \mid 0]$. Phase profiles of the $[4 \mid 0]$ state and $[3 \mid 0]$ state at $\gamma=0.3$ are shown in (b) and (c), respectively. ends up in different states-namely, in the state $\left[1+1_{1}\right.$ $\left.+1_{2} \mid 0\right]$ for $\gamma_{0}=0.3$ or in the state $\left[1+1_{1}+1_{1} \mid 0\right]=[3 \mid 0]$ for $\gamma_{0}=0.35$. The IVCs of both states are shown in Fig. 4 . The profiles of the phase gradients are shown in Fig. 4(c).

Our attempts to construct the states with a higher number of bunched fluxons, e.g., $[4+4 \mid 0]$, have failed since four fluxons do not fit into one well. We have concluded that such states immediately get converted into one of the lower-order states.

\section{Three coupled junctions}

We have performed numerical simulation of Eqs. (3) and (4), using the same technique as described in the previous section. Our intention here is to study the three-junction case in which the fluxon is put in the middle junction $([0|1| 0]$ state). All other parameters were the same as in the case of the two-junction system, except for the ratio of the critical currents $J$, which was taken equal to 1 . This simplest choice is made because in a system of $N>2$ coupled identical junctions Cherenkov radiation appears in a $[0|\cdots| 0|1| 0|\cdots| 0]$ state for $u>\bar{c}_{-} \approx 0.765$ (this pertains to $S=-0.5)$.

Figure 5 shows the IVCs of the original state $[0|1| 0]$, as well as IVCs of the bunched state $[0|1+1| 0]$, for $M$

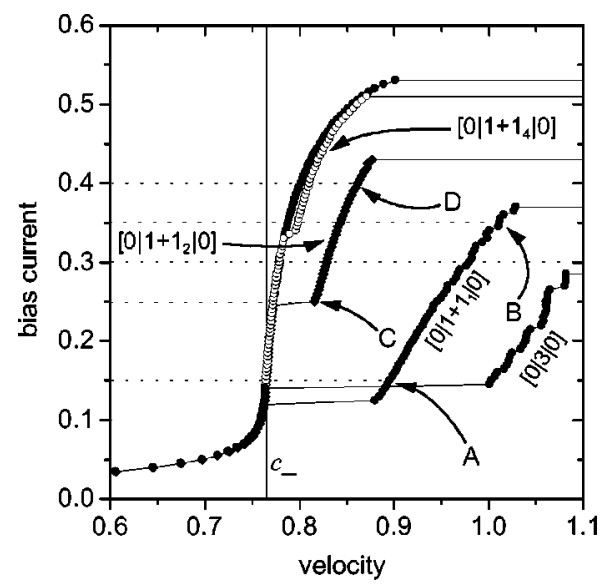

FIG. 5. Current-velocity characteristics of the state $[0|1| 0]$, bunched state $\left[0\left|1+1_{M}\right| 0\right]$ for three different cases, $M=1,2,3$, and the state $[0|3| 0]$. The profiles of the Josephson phase gradients at points $A-D$ are shown in Fig. 6
$=1,2,3$. The profiles of the phase gradients at points $A-D$ are shown in Fig. 6. Qualitatively, bunching in the threefold system takes place in a similar fashion as that in the twofold system. Nevertheless, we did not succeed in creating a stable fluxon configuration with $M=3$, although stable states with other $M$ were obtained. We would like to mention that when the second fluxon was put in the second minimum of the potential to get a state with $M=2$, the state with $M=1$ has been finally established as a result of relaxation. The same behavior was observed when we put the fluxon initially in the third minimum; the system ended up in the state [1 $\left.+1_{2} \mid 0\right]$. For $M \geqslant 4$, the behavior was as usual. We tried to vary $\Delta x$ smoothly, so that the center of the trailing fluxon would correspond to different positions between the second and fourth wells, but in this case we did not succeed in

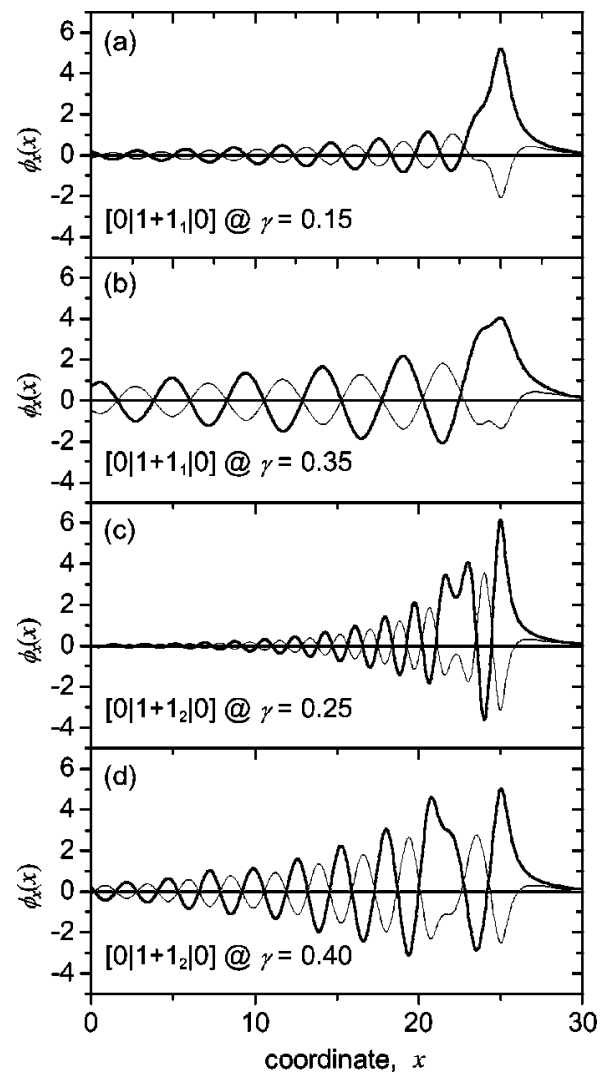

FIG. 6. The profiles of the Josephson phase gradients $\phi_{x}^{A, B}(x)$ in $\left[0\left|1+1_{M}\right| 0\right]$ states at points $A-D$ marked in Fig. 5 . 
obtaining the $\left[1+1_{3} \mid 0\right]$ state.

Following the same approach as for two coupled junctions, we tried to construct $[0+1|1| 0+1]$ states. As in the case $N=2$, these states were found to be unstable for any $M>0$; e.g., they would split into $\left[0\left|1+1_{2}\right| 0\right]$ and $[1 \mid$ $-1 \mid 1]$. The state $[0|2+2| 0]$ was not stable either for $M$ $=1,2,3$ and the bias currents $\gamma_{0}=0.20,0.30,0.35$.

The state $[0|2+1| 0]=[0|3| 0]$, constructed by combining solutions for the $[0|1| 0]$ and $[0|2| 0]$ states moving with equal velocities, was found to be stable when starting at $\gamma$ $=0.25$ and sweeping the bias current up and down. The dependence $u(\gamma)$ is shown in Fig. 5. One may note that for the states $[0|2| 0]$ and $[0|3| 0]$ the dependence is not smooth. Indeed, for these states the Cherenkov radiation tail is so long $(\sim L)$, that our annular system cannot simulate an infinitely long system, resulting in Cherenkov resonances which inevitably appear in the system of a finite perimeter. 6,7

\section{ANALYSIS AND DISCUSSION}

Because of the nonlinear nature of the bunching problem, it is hardly tractable analytically. Therefore, we here present an approach in which we analyze the asymptotic behavior of the fluxon's front and trailing tails in the linear approximation. This technique is similar to that employed in Ref. 9. We assume that, at distances which are large enough in comparison with the fluxon's size, the fluxon's profile is exponentially decaying,

$$
\phi(x, t) \propto \exp [p(x-u t)],
$$

where $p$ is a complex number which can be found by substituting this expression into Eqs. (1) and (2). As a result we arrive at the equation

$$
\left|\begin{array}{cc}
\frac{p^{2}}{1-S^{2}}-p^{2} u^{2}-1-\alpha p u & -\frac{S p^{2}}{1-S^{2}} \\
-\frac{S p^{2}}{1-S^{2}} & \frac{p^{2}}{1-S^{2}}-p^{2} u^{2}-\frac{1}{J}-\alpha p u
\end{array}\right|=0 .
$$

In general, this yields a fourth-order algebraic equation which always has four roots. If we want to describe a soliton moving from left to right with a radiation tail behind it, we have to find the values $p$ among the four roots which adequately describe the front and rear parts of the soliton. Because the front (right) part of the soliton is not oscillating, it is described by Eq. (11) with real $p<0$. The rear (left) part of the soliton is the oscillating tail; consequently it should be described by Eq. (11) with complex $p$ having $\operatorname{Re}(p)>0$, the period of oscillations being determined by the imaginary part of $p$. Analyzing the fourth-order equation, we conclude that the two necessary types of the roots coexist only for $u$ $>\bar{c}_{-}$, which is quite an obvious result.

To analyze the possibility of bunched state formation, we consider two fluxons situated at some distance from each other. We propose the following two conditions for the two fluxons to form a bunched state.

(i) Since nonoscillating tails result only in repulsion between fluxons, while the oscillating tail leads to mutual trapping, the condition

$$
\operatorname{Re}\left(p_{l}\right)<\left|p_{r}\right|
$$

can be imposed to secure bunching. Here $p_{l}$ is the root of Eq. (12) which describes the left (oscillating) tail of the leading (right) fluxon, and $p_{r}$ is the root of Eq. (12) which describes the right (nonoscillating) tail of the trailing (left) fluxon.

(ii) The relativistically contracted fluxon must fit into the minimum of the tail, i.e.,

$$
\frac{\pi}{\operatorname{Im}(p)}>\sqrt{\frac{u^{2}}{\bar{c}_{-}^{2}}-1},
$$

where $\pi / \operatorname{Im}(p)$ is half of the wavelength of the tail-forming radiation (the well's width), and the expression on the RHS of Eq. (14) approximately corresponds to the contraction of the fluxon at the trans-Swihart velocities. Although our system is not Lorentz invariant, numerical simulations show that the fluxon indeed shrinks (not up to zero) when approaching the Swihart velocity $\bar{c}_{-}$from both sides.

Following this approach, we have found that the second condition (14) is always satisfied. The first condition (13) gives the following result. Bunching is possible at $u>u_{b}$ $>\bar{c}_{-}$. The value of $u_{b}$ can be calculated numerically, and for $S=-0.5, J=0.5$, and $\alpha=0.04$ it is $u_{b}=0.837$. Looking at Fig. 2, we see that this velocity corresponds to the bias point where the $\left[1+1_{M} \mid 0\right]$ states cease to exist. Thus, our crude approximation reasonably predicts the velocity range where the bunching is possible.

\section{CONCLUSION}

In this work we have shown by means of numerical simulations the following.

(i) The emission of the Cherenkov plasma waves by a fluxon moving with high velocity creates an effective potential with many wells, where other fluxons can be trapped. This mechanism leads to bunching between fluxons of the same polarity.

(ii) We have proved numerically that in the system of two and three coupled junctions the bunched states for the fluxons in the same junction such as $[1+1 \mid 0],[1+2 \mid 0]$, $[2+2 \mid 0]$, and $[0|1+1| 0]$ are stable. The states with fluxons in different junctions like $[1 \mid 0+1]$ and $[0+1|1| 0+1]$ are numerically found to be unstable (except for the degenerated case $M=0$, when [1|1] is a simple in-phase state).

(iii) Bunched fluxons propagate at a substantially higher velocity than the corresponding free ones at the same bias current, because of lower losses per fluxon.

(iv) When decreasing the bias current, transitions between the bunched states with different separations between fluxons were not found. This behavior differs from what is known about bunched states in a discrete system. ${ }^{9}$ In addition, a splitting of multifluxon states into the states with smaller numbers of bunched fluxons is observed.

\section{ACKNOWLEDGMENT}

This work was supported by Grant No. G0464-247.07/95 from the German-Israeli Foundation. 
*Electronic address: e.goldobin@fz-juelich.de, homepage: http:// www.geocities.com/e_goldobin

†Electronic address: malomed@eng.tau.ac.il

${ }^{1}$ R. Kleiner, F. Steinmeyer, G. Kunkel, and P. Müller, Phys. Rev. Lett. 68, 2394 (1992); R. Kleiner and P. Müller, Phys. Rev. B 49, 1327 (1994).

${ }^{2}$ R. Kleiner, P. Müller, H. Kohlstedt, N.F. Pedersen, and S. Sakai, Phys. Rev. B 73, 3942 (1994).

${ }^{3}$ P. Müller, in Festkörperprobleme Advances in Solid State Physics, edited by Helbig (Vieweg, Braunschweig, 1995), Vol. 34, p. 1.

${ }^{4}$ A. V. Ustinov, in Physics and Materials Science of Vortex States, Flux Pinning and Dynamics, Vol. 356 of NATO Advanced Study Institute, Series $E$, edited by R. Kossowsky et al. (Kluwer Academic, Dordrecht, 1999), pp. 465-488.

${ }^{5}$ Yu.S. Kivshar and B.A. Malomed, Phys. Rev. B 37, 9325 (1988).

${ }^{6}$ E. Goldobin, A. Wallraff, N. Thyssen, and A.V. Ustinov, Phys. Rev. B 57, 130 (1998).
${ }^{7}$ E. Goldobin, A. Wallraff, and A. V. Ustinov, J. Low Temp. Phys. 119, 589 (2000); cond-mat/9910234 (unpublished).

${ }^{8}$ G. Hechtfischer, R. Kleiner, A.V. Ustinov, and P. Müller, Phys. Rev. Lett. 79, 1365 (1997).

${ }^{9}$ A.V. Ustinov, B.A. Malomed, and S. Sakai, Phys. Rev. B 57, 11 691 (1998).

${ }^{10}$ S. Sakai, Phys. Rev. B 36, 812 (1987).

${ }^{11}$ B.A. Malomed, Phys. Rev. B 47, 1111 (1993).

${ }^{12}$ I.V. Vernik, N. Lazarides, M.P. Sørensenl, A.V. Ustinov, N.F. Pedersen, and V.A. Oboznov, J. Appl. Phys. 79, 7854 (1996).

${ }^{13}$ S. Sakai, P. Bodin, and N.F. Pedersen. J. Appl. Phys. 73, 2411 (1993).

${ }^{14}$ E. Goldobin, A. Golubov, and A.V. Ustinov, Czech. J. Phys. 46, 663 (1996), LT-21 Suppl. S2.

${ }^{15}$ E. Goldobin, available online: http://www.geocities.com/ SiliconValley/Heights/7318/StkJJ.htm, 1999.

${ }^{16}$ N. Grønbech-Jensen, D. Cai, and M.R. Samuelsen, Phys. Rev. B 48, 16160 (1993). 